\title{
Comparison of endoscopy, histology, and cytokine mRNA of the equine gastric mucosa
}

\author{
M. Pietra • M. Morini • G. Perfetti • A. Spadari • \\ P. Vigo - A. Peli
}

Published online: 15 May 2010

(C) Springer Science+Business Media B.V. 2010

\begin{abstract}
In recent years, gastric ulceration has been recognized as a common, possibly performancelimiting disease, of adult horses. The aim of this study was to compare endoscopic features, histological diagnosis, and mRNA levels of various cytokines (TNF- $\alpha$, IL-1 $\beta$, and IL-13) from horse gastric biopsies. Eleven horses suffering from equine gastric ulcer syndrome and seven horses with normal histological gastric features were assessed. No correlation between endoscopic features and histology (i.e., the gold standard) was observed. Based on histological diagnosis, a significant $(p<0.05)$ increase in cytokine mRNA levels (specifically, TNF- $\alpha$ and IL-13) was observed in horses affected by equine ulcerative gastric syndrome.
\end{abstract}

Keywords Horse · Cytokine · Histopathology · Equine gastric ulcer syndrome

\author{
Abbreviations \\ IL-1 $\beta \quad$ interleukin-1 $\beta$ \\ IL-13 interleukin-13 \\ TNF- $\alpha$ tumour necrosis factor- $\alpha$
}

\section{Introduction}

Equine gastric ulcer syndrome is a disease involving up to $80 \%$ of racehorses (Begg and O'Sullivan 2003); it is often underdiagnosed because of non-specific symptoms and a lack of markers for laboratory diagnosis. In particular, it may show clinical symptoms similar to colic, such as yawning, bruxism, anorexia, and weight loss, or more frequently, it may

M. Pietra $(\bowtie) \cdot$ G. Perfetti $\cdot$ A. Spadari $\cdot$ A. Peli

Dept. of Veterinary Clinical Sciences, Alma Mater Studiorum - University of Bologna, Bologna, Italy e-mail: marco.pietra@unibo.it

M. Morini · P. Vigo

Dept. of Veterinary Public Health and Animal Pathology, Alma Mater Studiorum -

University of Bologna, Bologna, Italy 
manifest itself by a reduction in performance (Bell et al. 2007). The most specific diagnostic procedure currently used consists of gastroscopy and subsequent histological examination of biopsy samples.

To diagnose gastric ulcer syndrome, we analyzed both healthy subjects and those with gastropathy, we compared endoscopic findings with histological findings, and analyzed cytokine mRNA from gastric tissue.

In this study, we set out to explore certain cytokines that are known potent immunomodulators with pro-inflammatory actions. In the absence of studies on cytokine panels in the stomachs of normal horses and horses suffering from gastritis, we selected cytokines [interleukin (IL)-1 $\beta$ (IL-1 $\beta$ ), tumour necrosis factor- $\alpha$ (TNF- $\alpha$ ), and IL-13] that are involved in both $\mathrm{Th} 1$ and $\mathrm{Th} 2$ responses.

\section{Materials and methods}

Eighteen adult horses were studied at the Veterinary Clinical Department of Alma Mater Studiorum - University of Bologna between January 2006 and December 2008. All either showed clinical signs similar to recurrent colic syndrome or demonstrated a decrease in performance not justified by other pathologies. The subjects underwent gastroscopy (Pentax VSB-3440) after an 8-h fast and sedation (detomidine and butorphanol). In all subjects, multiple samplings from the same gastric areas were taken for histological examination and the analysis of cytokine mRNA.

The samples for cytokine analysis were placed into cuvettes, immediately frozen, and kept at a temperature of $-80^{\circ} \mathrm{C}$ until cytokine analyses was carried out ( $\beta$-actin was used as a housekeeping control to monitor the correct extraction procedure). Both the cytokine primers and cycle parameters were uniquely designed and tested on horse lymphocytes stimulated with concanavalin A.

Specifically, we extracted RNA from biopsy specimens using an RNeasy Mini Kit from Qiagen. The extracted RNA was then treated with DNase and subsequently reversetranscribed into DNA by reverse transcriptase. The cDNA obtained was then amplified by PCR and analyzed by electrophoresis.

The samples for histological examination were positioned by means of special paper (Bio-optica), fixed in buffered formalin, embedded in paraffin, sectioned (4 $\mu \mathrm{m}$ thick), and stained with hematoxylin-eosin.

The results for the individual cytokines were expressed with the following values: (1) cytokine not present or (2) cytokine present. Furthermore, to allow subsequent statistical analysis, the results of endoscopy and the histological findings were also categorized according to the following schedule: (1) normal findings and (2) different degrees of gastritis. The results of the endoscopic and histological examinations were compared, respectively, with the cytokine results using the Chi square test, and a possible correlation between the histological and endoscopic results was analyzed using the Spearman Correlation Order Test. Significance was set at $p<0.05$.

\section{Results}

In 6 of 18 subjects examined, the endoscopic findings of the gastric cavity were normal. In the remaining cases, an ulcerative syndrome varying from mild to severe was diagnosed. Subsequent histological examination of the biopsy specimens (the gold standard) allowed the identification of seven subjects classified as normal and 11 that suffered from varying 
degrees of gastritis. The correlation between the endoscopic and histological findings was not significant $(p=0.09)$. Regarding the analysis of the cytokines, using the histological findings as the gold standard, in healthy subjects, TNF- $\alpha$ was seen in 1 of 7 samples, IL-1 $\beta$ in 3 of 7 samples, and IL-13 in no samples, while in subjects with gastritis, TNF- $\alpha$ was found in 8 of 11 samples, IL-1 $\beta$ in 9 of 11 samples, and IL-13 in 5 of 11 samples.

Using the Chi-square test to compare each cytokine found in each group (healthy horses $v s$. horses suffering from gastritis as diagnosed histologically), the results showed a significant presence of mRNA for TNF- $\alpha$ and IL-13 in patients with gastritis, while no statistical differences were observed for IL- $1 \beta$ mRNA between healthy subjects and those with gastritis. Repeating the comparison between the cytokines in horses classified endoscopically as healthy or suffering from ulcerative syndrome, the Chi-square tests detected a statistically significant difference between the two groups only for TNF- $\alpha$ mRNA.

\section{Discussion}

The study of cytokines in animal tissue is still in its infancy as shown by the few references that refer to the analysis of tissue cytokines of organs other than the stomach or in species other than horses. In particular, the main studies found in the veterinary literature on the determination of tissue cytokines include the analysis of cytokines extracted from bronchial biopsies of horses suffering from recurrent airway obstruction (Ainsworth et al. 2006; Riihimäki et al. 2008); from the intestinal walls of dogs and cats with inflammatory bowel disease (De Majo et al. 2008; Janeczko et al. 2008); and, finally, from the intestinal mucosa of pigs (Andersson et al. 2007).

The purpose of this study was, therefore, to contribute to the knowledge of the cytokines that are activated in the process of equine gastric ulcer syndrome by comparing the data obtained in the course of gastritis with the cytokine mRNAs derived from gastric biopsies of healthy subjects. It is necessary to emphasize that our study refers almost exclusively to the mucosal layer as our biopsies, endoscopically performed, include only the most superficial portions of the gastric wall. The first objective was to design and test the primers needed to identify TNF- $\alpha$, IL-1 $\beta$, and IL-13 mRNAs in gastric mucosal tissue. Although limited in number, the choice of these three cytokines allowed an analysis of the Th1 and $\mathrm{Th} 2$ responses while identifying both the early stages of inflammation (as evidenced by an increase in IL-1 $\beta$ ) and late responses (increases in both TNF- $\alpha$ and IL-13). It is interesting to observe the significant increase in TNF$\alpha$ and IL-13 during the development of equine gastric ulcer syndrome that can, if confirmed on a larger sample of patients, be considered a specific marker for gastritis. Moreover, we emphasize the absence of any correlation $(p=0.09)$ between the endoscopic and histological findings, which could also have been the result of using a non-parametric test.

In conclusion, this study provides valuable insights into a preliminary population of cytokines in the gastric mucosa of horses suffering from gastric ulcer syndrome; however, more studies using an increased number of animals and the use of quantitative PCR in place of qualitative PCR are necessary.

\section{References}

Ainsworth DM, Wagner B, Franchini M, Grünig G, Erb HN, Tan J-T (2006) Time-dependent alterations in gene expression of interleukin-8 in the bronchial epithelium of horses with recurrent airway obstruction. Am J Vet Res 67:669-677 
Andersson M, Berg M, Fossum C, Jensen-Waern M (2007) Development of a microarray for studying porcine cytokine production in blood mononuclear cells and intestinal biopsies. J Vet Med A 54:161168

Begg LM, O’Sullivan CB (2003) The prevalence and distribution of gastric ulceration in 345 racehorses. Aust Vet J 81:199-201

Bell RJW, Mogg TD, Kingston JK (2007) Equine gastric ulcer syndrome in adult horses. N Z Vet J 55:1-12

De Majo M, Pugliese M, Galia S, Mazzullo G, La Camera E, Fera MT (2008) Cytokine mRNA quantification in gastro-intestinal biopsies of dogs with idiopathic chronic enteropathies by Real Time RT-PCR: preliminary results. Vet Res Commun 32(Suppl 1):275-277

Janeczko S, Atwater D, Bogel E, Greiter-Wilke A, Gerold A, Baumgart M, Bender H, McDonough PL, McDonough SP, Goldstein RE, Simpson KW (2008) The relationship of mucosal bacteria to duodenal histopathology, cytokine mRNA, and clinical disease activity in cats with inflammatory bowel disease. Vet Microbiol 128:178-193

Riihimäki M, Raine A, Pourazar J, Sandström T, Art T, Lekeux P, Couëtil L, Pringle J (2008) Epithelial expression of mRNA and protein for IL-6, IL-10 and TNF- $\alpha$ in endobronchial biopsies in horses with recurrent airway obstruction. BMC Vet Res $4: 8$ 